\title{
Profiling outpatient workload: practice variations between consultant firms and hospitals in south west England
}

\author{
Alex C Faulkner, Ian M Harvey, Tim J Peters, Deborah J Sharp, Stephen J Frankel
}

\begin{abstract}
Objectives-To describe the variation in outpatient new to old ratios between consultants and between providers for seven high volume specialties (four surgical, three medical).

Design-This was a descriptive study at consultant and provider unit level based upon patient administration system data from the South and West Regional Health Authority for the financial year 1992-93. Additional components of variance analysis was used to distinguish individual consultant effects from host institution effects.
\end{abstract}

Setting-The former South Western Regional Health Authority area from Gloucestershire to Cornwall.

Subjects-Altogether 345 consultant firms in seven specialties grouped into 13 provider unit groups.

Main measures-New to old ratio, omitting elective inpatients followed up as outpatients.

Results-Variation between consultants is greater in surgical than in medical specialties, while absolute levels of new to old ratios tend to be higher in surgical specialties than in medical. Variation between provider unit groups is also greater in surgical specialties. Analysis of variance shows that more total variance is attributable to provider unit group in gynaecology than in other specialties.

Conclusions-Within individual specialties there is evidence of substantial variation that is not attributable to underlying differences in morbidity patterns. There is evidence of marked variation in terms of both individual consultants and institutions, a finding that provides the springboard for further analytical work. Published routine outpatient activity statistics should distinguish between new referrals, inpatient follow up, and clinic rebookings.

Department of Social Medicine, University of Bristol, Canynge Hall, Whiteladies Road, Bristol BS8 2PR A C Faulkner I M Harvey T J Peters D J Sharp

S J Frankel

Correspondence to: Mr A C Faulkner.

Accepted for publication September 1996

\section{(7 Epidemiol Community Health 1997;51:310-314)}

The importance of outpatient services in health services policy is signalled by the UK Patient's Charter waiting time targets. Service rate variations are evident in many aspects of outpatient services. ${ }^{1}$ A recent study has drawn attention to historical trends in total outpatient workload and in workload per consultant in the UK, ${ }^{2}$ suggesting that while the total number of hos- pital consultants has increased over the last 40 years, numbers of both follow up and new patients seen per consultant have declined, leading overall to relatively little change in the total number of outpatients seen. The nature of the historical "outpatient problem" is changing as more flexible and complex models of care delivery are being introduced at the primary/ secondary care interface. ${ }^{3}$ Further examination of the processes of outpatient care delivery in the National Health Service is therefore timely. Routine workload activity data now available can be disaggregated to examine separately the different reasons for outpatient attendance within specialties and within provider unit groups. ${ }^{45}$

One approach to the problems of outpatient workloads and waiting lists is to examine the extent to which patients are recycled in outpatient clinics. ${ }^{267}$ It can be assumed that a reduction in re-attendances within outpatient clinics frees time in which, in principle, additional new patients may be seen. This is indeed one reason for calls to increase the proportions of consultations conducted by consultants, who are widely assumed to be less inclined to recycle patients than are junior staff. $^{8}$ It is also part of the rationale for the Patients' Charter requirement that all newly referred patients be seen by a consultant. Also, the value of routine outpatient surveillance for a widening range of conditions is under scrutiny-for example, asthma, hypertension, rheumatoid arthritis, routine surgical follow up for non-complicated cases, and follow up of treated malignant conditions such as breast cancer. ${ }^{9}$

The ratio of new to established ("old") patients provides a measure of recycling. Variation in this ratio has not previously been documented at a hospital, specialty or consultant level. Demonstration of substantial variation beyond that expected from casemix differences, provides prima facie evidence of variation in clinical judgement and practices between individuals and institutions. This can be a springboard for further analytical work ultimately of benefit to patients. ${ }^{10}$ For example, studies of inpatient practice variations in the surgical management of benign prostatic hypertrophy, ${ }^{11}$ led to the identification of a critical level of variation, stimulating trials of the effectiveness of alternative therapies.

The aims of the present study are to describe the variation in the ratio of new referrals to re-attendances in the outpatient workload at various levels of aggregation, to estimate the 


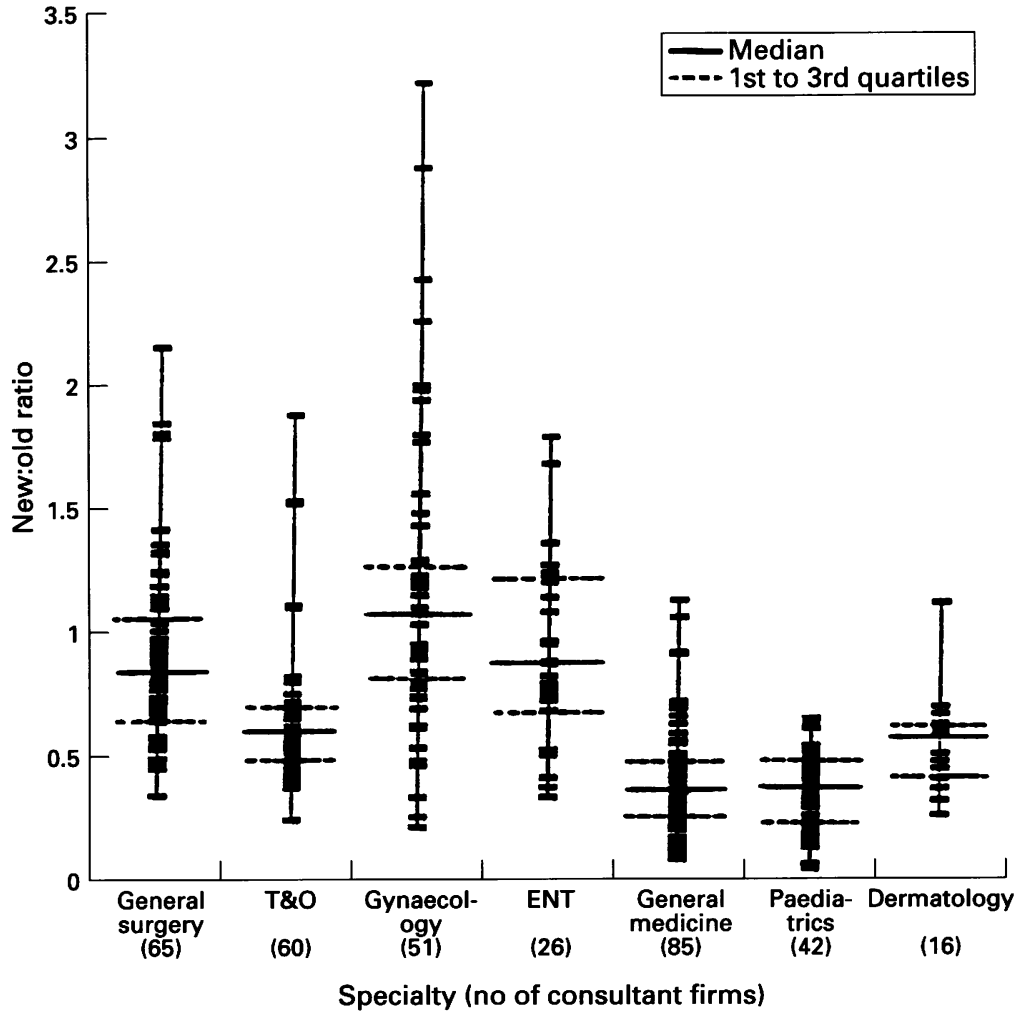

Figure 1 Variation in new to old referral ratios for outpatients in relation to consultant specialties. attendance)". The figures for total new referrals seen were generated from combining the following referral categories: "GP referral", "A \& E referral", "Referral by other consultant", "Self referral", "Private patient (same consultant)", "Domiciliary visit (same consultant)", "Outpatient attendance, other purchaser" and "Outpatient attendance, after emergency admission". Old (established) patient numbers were obtained from a single code, "Outpatient attendance, this provider" (defined as attendances initiated in the consultant clinic in which the patient is again being seen). Inpatients admitted electively and subsequently followed up in outpatients were excluded from the calculation of the ratio, since their attendance represents a distinct element of outpatient workload, separate both from rebooked attendances and from new referrals. Our new to old ratio differs therefore from the new to follow up ratio which can be calculated from the KH09 returns. Except for inpatient follow up, these classifications to new or follow up adopt the conventions used to derive the Körner new and total follow up figures submitted by regional health authorities to the Department of Health.

Data were collected for all consultants within the former south western region from the specialties of general surgery, general medicine, trauma and orthopaedics (T\&O), paediatrics, gynaecology, ear nose and throat (ENT), and dermatology for the financial year 1992-93, the most recent complete year available. These specialties were selected because they are high volume specialties spanning all ages and representing both surgical and non-surgical disciplines. Consultant codes for which fewer than 200 consultations were recorded for the whole year were excluded to avoid random variation due to small numbers.

Hospitals have been grouped together into "provider unit groups" to reflect the locations in which individual consultants predominantly or exclusively work. In cases where consultants worked in more than one provider unit group, the unit where most cases were seen was deemed to be the consultant's base for the purposes of analysis.

\section{STATISTICAL METHODS}

Interpretation of the new to old ratio may be aided by recognising that it can be defined as: $1 /$ mean no of re-attendances for each new patient

Any given ratio may therefore be alternatively expressed as the number of re-attendances associated on average with each new referral attendance (in other words, the mean re-attendance). For example, a new to old ratio of 2.00 is equivalent to a mean re-attendance of 0.50 .

Following descriptive analyses, the proportion of the variance in either the ratio or the mean re-attendance attributable respectively to the individual consultant/firm and to the provider unit group was assessed using a components of variance analysis ${ }^{12}$ in Minitab for Windows. Prior to undertaking these analyses of variance, the validity of the assumption of a 


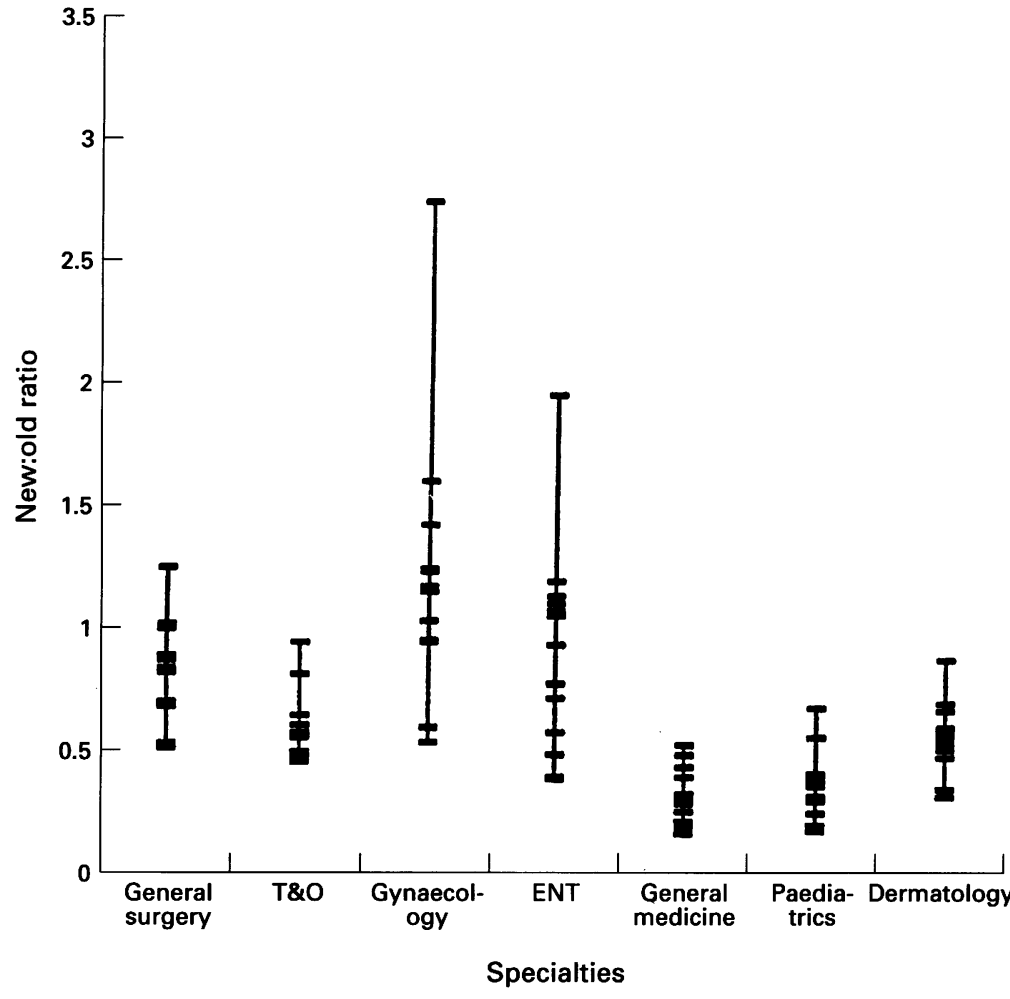

Figure 2 Variation in new:old ratios for outpatients between 13 provider groups within seven specialties.

Table 1 Ratios of new referrals seen to re-attendances initiated in the consultant clinic, south western region, 1992-93 (ranges, medians, and quartiles)

\begin{tabular}{|c|c|c|c|c|c|c|}
\hline \multirow[b]{2}{*}{ Specialty } & \multirow{2}{*}{$\begin{array}{l}\text { No of } \\
\text { consultants/firms }\end{array}$} & \multirow[b]{2}{*}{ Range } & \multicolumn{4}{|c|}{ Quartiles } \\
\hline & & & $1 s t$ & Median & $3 n d$ & $Q^{*}$ \\
\hline General surgery & $(65)$ & $0.33-2.15$ & 0.65 & 0.81 & 1.04 & 0.20 \\
\hline Trauma and orthopaedics & $(60)$ & $0.23-1.87$ & 0.47 & 0.57 & 0.67 & 0.10 \\
\hline Gynaecology & (51) & $0.20-3.21$ & 0.78 & 1.06 & 1.27 & 0.25 \\
\hline ENT & (26) & $0.32-1.78$ & 0.67 & 0.84 & 1.19 & 0.26 \\
\hline General medicine & $(85)$ & $0.07-1.12$ & 0.23 & 0.30 & 0.44 & 0.11 \\
\hline Paediatrics & $(42)$ & $0.03-0.64$ & 0.20 & 0.32 & 0.45 & 0.13 \\
\hline Dermatology & (16) & $0.25-1.11$ & 0.40 & 0.58 & 0.60 & 0.10 \\
\hline
\end{tabular}

$* \mathrm{Q}$ (quartile deviation) $=\frac{\text { quartile } 3-\text { quartile } 1^{14}}{2}$

Table 2 Magnitudes of absolute and inter-consultant firm variance in new:old ratios

\begin{tabular}{lllll}
\hline Specialty & $\begin{array}{l}\text { No of } \\
\text { consultants/ } \\
\text { firms }\end{array}$ & $\begin{array}{l}\text { Absolute inter- } \\
\text { consultant } \\
\text { variance }\end{array}$ & $\begin{array}{l}\text { Absolute inter- } \\
\text { provider unit } \\
\text { group variance }\end{array}$ & $\begin{array}{l}\text { Total } \\
\text { variance }\end{array}$ \\
\hline General surgery & $(65)$ & 0.110 & 0.024 & 0.134 \\
Trauma and orthopaedics & $(60)$ & 0.066 & 0.014 & 0.080 \\
Gynaecology & $(49)^{*}$ & 0.143 & 0.113 & 0.256 \\
ENT & $(26)$ & 0.078 & 0.071 & 0.149 \\
General medicine & $(85)$ & 0.033 & 0.012 & 0.045 \\
Paediatrics & $(42)$ & 0.022 & 0.003 & 0.025 \\
Dermatology & $(15) \dagger$ & 0.013 & 0.005 & 0.018 \\
\hline
\end{tabular}

* Excluding two outliers; $\uparrow$ excluding one outlier.

Gaussian distribution was assessed by the use of normal plots and the Shapiro-Wilk test. ${ }^{13}$ For all specialties other than general surgery and $\mathrm{T} \& \mathrm{O}$, the new to old ratio was a closer fit to a Gaussian distribution than was the mean re-attendance, although for dermatology and gynaecology it was necessary to exclude one and two (very high) outliers respectively. For general surgery and $\mathrm{T} \& \mathrm{O}$, the results of the analysis of variance for the new to old ratio were nevertheless very similar to those for the mean re-attendance and hence for simplicity the results for all specialties are presented for the new to old ratio.

Given the different numbers of consultations involved for each consultant firm within a given specialty, any such components of variance models should account for the resultant differential random variation. To achieve this, as well as the unweighted analysis of new to old ratios, two further approaches were adopted. The first was an analysis of the new to old ratios using weights in proportion to the total numbers of new plus old consultations (these were heuristic weights given that no simple equation exists for the variance of such ratios). The second analysis was of the number of new consultations as a proportion $(p)$ of the total new plus old consultations $(N)$, using weights proportional to the inverse of the variance of the proportion $\mathrm{p}$ (assuming a binomial distribution these variances were $p(1-p) / N)$. These analyses are presented in table 3.

\section{Results}

VARIATION BY CONSULTANT FIRM

The main finding is the existence of marked variation between consultant firms within specialties in the ratio of new referrals to re-attendances (fig 1 and table 1). The greatest variation is seen in gynaecology, ENT, and general surgery, and the greatest overall range in gynaecology and general surgery. Relatively less variation was observed in $\mathrm{T} \& \mathrm{O}$, general medicine, paediatrics, and dermatology. These findings were not altered by exclusion of consultants based in the Bristol teaching hospitals whose outpatient practice might reflect the consequences of providing a tertiary referral service.

There was also notable variation between specialties in terms of the absolute level of new to old ratios as indicated by the medians in figure 1. General surgery, gynaecology, and ENT have relatively high ratios (lower mean re-attendances), and general medicine and paediatrics have relatively low ratios (higher mean re-attendances).

VARIATION BY PROVIDER UNIT GROUP

Since each of the provider unit groups in this study is providing a general service to a local population, casemix differences that may exist between consultants can be reduced by examining data relating to units rather than to individual consultants. A degree of variation clearly persists between provider units (fig 2), although as anticipated this is less than the variation between consultants. Absolute interunit variation varies across specialties with the non-surgical specialties exhibiting lower variation.

It is useful in addition to examine what proportion of the variation between consultants is attributable to the effect of the unit in which they work. The results of the components of variance models are presented in table 2 . Total variance is highest in gynaecology, ENT, and general surgery, consistent with the picture in figure 1 . Both the absolute inter-provider unit 
Table 3 Percentages of variance attributable to provider unit group, calculated by three different methods

\begin{tabular}{llll}
\hline & \multicolumn{4}{l}{ Percentage of variance attributable to provider unit group } \\
\cline { 2 - 4 } Specialty & New:old ratio* & New:old ratiot & Proportion new $\ddagger$ \\
\hline General surgery & 18.0 & 23.2 & 30.7 \\
Trauma and orthopaedics & 17.2 & 21.3 & 29.8 \\
Gynaecology & 44.1 & 46.2 & 55.4 \\
General medicine & 26.4 & 25.3 & 33.3 \\
Paediatrics & 13.6 & 21.9 & 25.5 \\
\hline
\end{tabular}

Excluding ENT and dermatology because of relatively small numbers of consultants/consultations. * Unweighted analysis; tweighted by total numbers of new and old outpatient consultations; $\ddagger$ weighted by the reciprocal of the variance of the proportion of new consultations (see Methods).

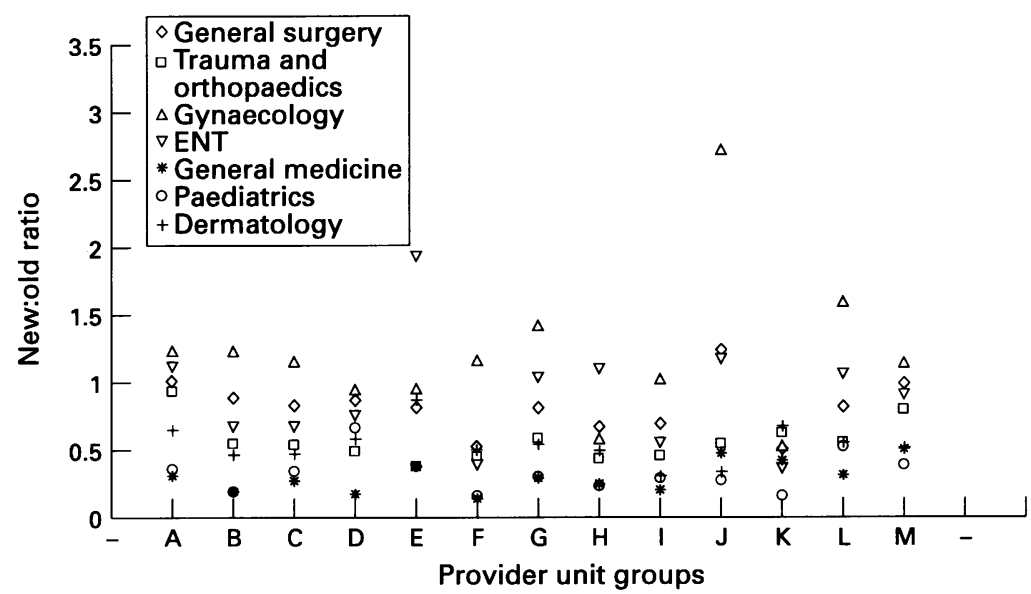

Figure 3 Variations in outpatient new:old ratios between seven specialties in 13 provider unit groups.

group variance ( 3 rd column table 2 ) and the proportion of total variance attributable to provider unit group are greatest in gynaecology (table 3). This is the case regardless of whether weighted or unweighted analysis of variance is performed.

The total numbers of consultations in ENT and dermatology are too small for reliable estimates to be made of the relative size of interprovider and intra-provider group variance. These specialties have been excluded from table 3.

Figure 3 displays the new to old ratios for all specialties in each provider unit group in the region. There is variation in the likelihood of re-attendance between the geographical areas represented by different provider unit groups, as shown for example by comparison of groups $\mathrm{K}$ and $\mathrm{L}$.

To assess the effect of the omission of elective inpatient follow up cases from the new to old ratios, these attendances were analysed as a proportion of total consultations for the three consultant data points at each extreme of the range in each specialty. This showed that for all but one specialty there was no clear difference in the proportion of total workload constituted by elective inpatient follow up between the two extremes. The exception was ENT, where consultant firms with the highest new to old ratios also had relatively high proportions of inpatient follow up in the workload $(8.1 \%$, $10.6 \%, 17.6 \%$ ), compared with those with the lowest new to old ratios, who conversely had consistently low proportions of inpatient follow up in their workloads $(0.7 \%, 4.1 \%, 0.3 \%)$.

\section{Discussion}

The new to old ratio investigated in this paper represents the balance between the two elements of outpatient workload which account for most patients seen. The variation in the profiles of inter-consultant and inter-provider ratios across specialties suggests strongly that a specialty-specific approach is essential in examining outpatient performance data. Of the seven specialties examined here, the four with the highest inter-consultant variance are surgical, and the three with the lowest variance are broadly medical. This pattern of variation seems specific to the outpatient setting. When, for example, standardised inpatient discharge ratios (defined as observed to expected numbers of discharges multiplied by 100) were examined, wider range and variation were seen in general medicine than in general surgery, ${ }^{15}$ the reverse of the pattern shown here for outpatients. This suggests that patients seen in the outpatient setting present distinctive problems of clinical management and organisation.

There are a number of possible reasons for this variation. One potential explanation is artefact due to data inaccuracy. However, the data demonstrate a reasonable and plausible level of consistency. This was seen, for example, in the relative magnitudes of new to old ratios in the surgical compared with non-surgical specialties-differences which make sense intuitively in terms of the higher proportions of chronic illness seen in the medical specialties. Hence in our view errors in data are unlikely to have had a substantial effect upon the overall profiles of variation found.

Some variation in the new to old ratio might be attributable to the classification of sub-specialisations. In gynaecology, for example, the consultants/firms with the higher ratios (seeing lower proportions of re-attendances) tended to be classified as "gynaecology only", rather than obstetrics and gynaecology combined. On the other hand, the general medicine data excluded recognised subspecialties such as cardiology, neurology, respiratory medicine, and gastroenterology. The persisting variation at provider unit group level shows that classification of subspecialties is not the full explanation of the variation, even in gynaecology.

Variation in the ratio might theoretically also be accounted for by different practice with regard to outpatient follow up of elective inpatients. The data do not in general show such an effect, except for ENT. In this specialty the effect seems to be that higher elective inpatient follow up is associated with a higher new to old ratio, suggesting that "recycling" of outpatients is inhibited by the relatively high proportions of follow up of inpatients in the ENT workload.

In summary, there is substantial variation which requires explanation. The components of variance analyses suggest that some variation is attributable to features of provider units and some to individual consultant behaviour. The absolute variation attributable to consultants is greater in surgical specialties than non-surgical specialties (table 2). A number of possible explanations can be suggested. Surgeons may in 
general pursue more independent, individual styles of practice than physicians due to differences in self selection into the specialties, education, or professional socialisation. Development of special interests within the surgical specialties may be greater than in the medical specialties. There was some suggestion that this was the case in gynaecology. A consultant with one of the lowest rates of new patient attendance had a particular interest in subfertility, investigation and management of which requires repeated visits. On the other hand, checking of a sample of the consultant firms in the other specialties at the extremes of the ranges of mean re-attendance did not suggest that generalist consultants (for example, general physicians) with particular specialist interests (for example, gastroenterology) tended to occupy the extremes. We conclude that this explanation does not apply generally.

A further possible explanation for inter-consultant variation is systematic variation in clinical judgement. It may be that the clinical knowledge base in the surgical specialties is less well rooted in clear evidence of effectiveness, is more complex, and is less amenable to standard-setting than is the case for medical specialties. There is certainly evidence from the south west to suggest that many more clinical guidelines have been developed in the medical than in the surgical specialties (D Baker, personal communication).

Effects at the level of the provider unit group are also substantial, the greatest being observed in gynaecology (table 2 and table 3 ). It is unlikely that this variation can be wholly attributed to underlying morbidity differences; the appropriate morbidity data are not available, but a study of factors predicting high mean re-attendance in another region in one specialty found that diagnosis and disease severity combined were able to account for only a small percentage $(<20 \%)$ of the variance found. ${ }^{20}$ In gynaecology, uncertain management of conditions such as menorrhagia and dysmenorrhoea may lead to hospital based conventions of practice. ${ }^{16}$ It is likely that institutional effects are operating. The hypothesis of a consultant "signature" 17 is not sufficient to account for the total variance found in these data; there are to some degree at least "institutional signatures" which are worthy of further examination.

The currently published routine data are generally unhelpful in analysing profiles of outpatient workloads. ${ }^{18}$ Measures specific to the complex dynamics of outpatient workloads are needed if a clear understanding of the reasons for variation in outpatient practice in different specialties is to develop. New data are not required to enable the crucial distinction to be drawn between the three key elements of outpatient workload: inpatient follow up, new referrals and clinic initiated re-attendances. These data are already available within the contract minimum data set for outpatient services. It would be straightforward to collate, present, and publish them in this form.

The existing research record has not systematically identified the key factors accounting for the high degrees of variation in the proportions of new referrals and recycled patients in outpatient workloads, though a number of contributory factors have been identified. ${ }^{1920} \mathrm{~A}$ complex array of factors is clearly involved. The systematic identification of these is the topic of a follow up study. The analysis presented here does not permit optimal follow up patterns to be inferred, but it does give new support to the view that substantial numbers of patients are attending unnecessarily or not frequently enough.

The authors thank Ms Jane Brooks of the South and West Regional Health Authority for assistance with extraction of the data analysed in this paper, and anonymous referees for comments on previous drafts of this paper.

Funding: the authors gratefully acknowledge the South and West Regional Research and Development Directorate for support for the research programme of which this study forms a part.

Conflicts of interest: none.

1 Faulkner A, Frankel S. Evaluation of delivery and use of outpatient services: a review of recent published evidence and developments with possible measures for action. Bristol: Health developments with possible measures for action. Bristol:

2 Armstrong D, Nicoll M. Consultants' workload in outpatient clinics. $B M \mathcal{F}$ 1995;310:581-82.

3 Frankel S, Faulkner A. The end of the outpatient problem? BMF 1994;309:1308.

4 Volans AP. Not all specialist groups hold outpatient clinics. BMF 1995;311:59.

5 Irvine $\mathrm{H}$, Scott $\mathrm{P}$. Consultants spend more time with each patient than in the past. $B M \mathcal{F} 1995 ; 311: 59$.

6 Anonymous. To come again, 3 months. Lancet 1976; 1168-9.

7 Roberts T, Heagerty AM, Foster P. Existing outpatien services could be more efficient. BMF 1995;311:60.

8 Griffiths RW. Plastic surgery outpatient audit: principles and practice of "consultant only" clinics. Br $\mathcal{F}$ Plast Surg 1990;43:735-41.

9 ONCOFEM/CNR-ACRO. Consensus conference on "Followup in breast cancer". ONCOFEM and CNR-ACRO, Bari, October 8th, 1994.

10 McPherson $\mathrm{K}$. Variations in hospitalisation rates: why and how to study them. In: Ham C, ed. Health care variations: assessing the evidence. London: King's Fund Institute, 1988: $15-20$.

11 Wennberg J. Practice variations and the need for outcomes research. In: Ham C, ed. Health care variations: assessing the evidence. London: King's Fund Institute, 1988:32-5.

12 Armitage P, Berry G. Statistical methods in medical research. Oxford: Blackwell Scientific Publications, 1987.

13 Altman DG. Practical statistics for medical research. London: Chapman \& Hall, 1991.

14 Blalock HM. Social statistics. New York: McGraw-Hill, 1972.

15 Price CE, Paul EA, Bevan RG, Holland WW. Equity and medical practice variation: relationships between standmedical practice variation: relationships between standardised discharge ratios in total and for selected condition 46:58-62.

16 Coulter A, Klassen A, Mackenzie IZ, McPherson K. Diagnostic dilatation and curettage: Is it used appropriately? nostic dilatation and

17 Wennberg J, Gittelsohn A. Variations in medical care among small areas. Scientific American 1982;246:100-12.

18 Government Statistical Service. Out-patients and ward attenders. Crown Copyright: Department of Health, 1991

19 Sullivan FM, Hoare TM. New referrals to rheumatology clinics-Why do they keep coming back? Br $\mathcal{F}$ Rheumatol 1990;29(1):53-7.

20 Sullivan FM, Hoare T, Gilmour H. Outpatient clinic referrals and their outcome. Br $\mathcal{F}$ Gen Pract 1992;42:111-5. 